it could be elicited by other agents using other mechanisms of cytotoxicity, is an open question.

Taken together these observations suggest several possibilities of considerable fundamental as well as clinical importance. (1) Immunological surveillance may well be a reality in the skin; (2) immunological surveillance in the skin may be suppressed by DNA-damaging agents; (3) if PUVA is both a promoter and an initiator, its longterm sequelae in man may be even more severe than has hitherto been suspected.

Finally, the results with PUVA must inevitably lead to an examination of the effects of naturally occurring psoralens present in some sun-tan preparations since the activity of these in sunshine must surely result in DNA damage to the skin (Ashwood-Smith Brit. med. J. 3 Nov. $1140 ; 1979)$.

\section{The case for layered convection}

\section{from Peter J. Smith}

INTEREST in the possibility of mantle-wide convection has revived recently, sparked off largely by the conclusion, derived most impressively by Cathles (The Viscosity of the Earth's Mantle, Princeton University Press, 1975), that contrary to previous widespread belief the viscosity of the mantle does not necessarily increase significantly with depth. With one of the most important restraints to the continuation of convection into the lower mantle removed, support for convection limited to, say, the asthenosphere is weakened, and a return to the earliest concepts of mantle-wide flow becomes possible.

Or does it? In a most thoughtful paper Richter (J. geophys. Res., 84, 6783; 1979) points out that viscosity is not the only criterion to be applied to the case for and against crust-to-core convection cells. Whatever the hypotheses about variation of viscosity in the mantle, it is an inescapable fact that earthquakes never occur below a depth of about $700 \mathrm{~km}$, implying strongly that in trench zones (where the deepest earthquakes occur) the subducting lithosphere cannot pass into the region below $700 \mathrm{~km}$. When an increase in viscosity with depth was accepted, this resistance to penetration seemed hardly surprising; but with that limitation removed, some other explanation of the barrier is required.

And the phenomenon is even more remarkable than a simple $700 \mathrm{~km}$ limit would suggest. From a new analysis of earthquakes in the Tonga-Kermadec subduction zone, Richter not only shows that all foci are shallower than $700 \mathrm{~km}$ but

Peter J. Smith is a Reader in the Department of Earth Sciences at the Open University. demonstrates (using focal mechanisms) that where shocks occur below $600 \mathrm{~km}$ the corresponding sections of the downgoing slab become subject to downdip compression. This confirms for a particular case a generalisation made earlier by Isacks and Molnar (Rev. Geophys. Space Phys., 9, 103; 1971) and strengthens support for the view that subducting lithosphere meets insurmountable resistances when approaching $700 \mathrm{~km}$ depths. So, indirectly, does Richter's discovery that the seismic energy released in the Tonga-Kermadec region decreases generally with depth but then increases phenomenally between 500 and $700 \mathrm{~km}$ that is, in the zone immediately above the seismic cutoff. The significance of this increase is that it more or less rules out nonbarrier explanations of the $700 \mathrm{~km}$ limit. It is possible to argue, for example, that subducting lithosphere does pass into the region below $700 \mathrm{~km}$ but that by then it has become sufficiently warm to deform by creep rather than brittle fracture, thereby eliminating earthquakes. But it is difficult to imagine a deep energy peak arising from a lithosphere gradually becoming hotter with depth. Similarly, it has been suggested that lithosphere passing through the $650+$ $\mathrm{km}$ zone changes phase, giving rise to a ductile high-density form incapable of supporting earthquake mechanisms. This proposal is more difficult to evaluate; but again the energy release peak at depth poses a severe problem.

So the $700 \mathrm{~km}$ limit must represent a real barrier to the passage of lithosphere, for the large energy release in the $500-700 \mathrm{~km}$ interval can only be explained in terms of large compressive stresses resulting from the approach towards an irresistible obstacle. What is that obstacle if it is not a viscosity increase? It could easily be chemical inhomogeneity, claims Richter, who envisages a chemically layered mantle with density increasing with depth from layer to layer. If this sounds implausible, the converse - a homogeneous mantle sounds even more implausible, at least as Richter explains it. If the mantle is homogeneous, he argues, it must have been homogeneous right from the start or it must have become so by thermal convective mixing. But mantle conditions are such that thermal mixing will only remove inhomogeneities of less than about $1 \%$. In other words, the conditions for the occurrence of a homogeneous mantle are more restrictive than those likely to give rise to a mantle with more than minimal inhomogeneities.

Convective systems with chemical density variations have been studied in considerable detail in seawater. Extending the principles thereby derived to the mantle, Richter concludes that a significantly inhomogeneous mantle will develop layering in which convection is restricted to the discrete layers. Precisely how many convective layers there are in the mantle will depend on how the Earth accreted and how the mantle developed originally as the residue left by the removal of crustal and core materials - and these factors are uncertain. But since the mantle is more likely to be inhomogeneous than homogeneous, it is more likely to have more than one convective layer than it is to have just one. So, although whole-mantle convection is a plausible option again thanks to revised views about the mantle's viscosity, the details of the convection could be rather different from those envisaged by the early supporters of the idea.

\section{Antigenic shift and drift}

\section{from a Correspondent}

INFLUENZA has been relatively dormant as a human disease for the past several years. However with the advent of recombinant DNA technology and of monoclonal antibodies, work on the influenza virus has burgeoned. Particularly exciting are the recent advances in knowledge of the structure of the viral haemagglutin (HA) and of the mechanisms which allow its antigenic variation and the consequent emergence of new viral strains. So it was not surprising to find that this molecule was of central interest at a workshop* held early in December near Canberra, Australia.

HA, the major glycoprotein of the viral envelope (molecular weight 75,000 ) is coded by an mRNA specified by one of the eight segments of RNA present in the virus particle. Pandemics of influenza occur in the human population when a major shift in the antigenic structure of HA allows the virus to infect people who are immune to previously circulating strains. Obviously one would like to compare the complete primary amino acid sequence of HAs of several different pandemic strains. However, despite considerable effort by the protein chemists (M. Waterfield, ICRF; C. Ward and T. Dopheide, CSIRO, Melbourne) such information has proved difficult and laborious to obtain. Consequently the nature of the variation involved in the creation of new pandemic strains has remained unknown. As reported at the workshop, several groups now have cloned in prokaryotic vectors, full length cDNA copies of the HA genes from viruses belonging to two of the three major pandemic groups. Complete DNA sequences have been established for the Asian $\mathrm{H}_{2}$ strain, which first emerged in 1957 (M-J. Gething, ICRF); for several members of the Hong Kong series which first appeared in 1967 (N. Sleigh \& G. Both, CSIRO, Sydney; W. Min Jou, Ghent; G. Threlfall, Searle) and for the Rostock *An International Workshop on 'Structure and Variation in Influenza Virus', organised by Graeme Laver and Gillian Air Australian National University, Canberra, Australia. 